Romy Hecht

\section{Trazado, paisaje y territorio: Cerro Sombrero y la arquitectura del petróleo en Magallanes ${ }^{1}$}

\section{Este artículo presenta al campamento petrolero de Cerro} Sombrero como un patrón fundacional en Magallanes; patrón no desde el punto de vista de ocupar el territorio en base a la repetición de un modelo formal, sino como un orden deliberado e impuesto a un territorio que, como veremos, no otorga señales de ocupación. A la larga, un rescate patrimonial de la construcción de arquitectura moderna más al sur del mundo.

This article seeks to bighlight and analyse Cerro Sombrero as a foundation pattern in Magallanes, not in the sense of occupying a territory by a repeated formal model. Cerro Sombrero shows an imposed and deliberated order in a territory without signals nor possibilities of occupation. At the end, the rescue of the southernmost modern architecture of the world.
Históricamente Magallanes fue el último trozo de Chile en ser ocupado no sólo por su clima y lejanía, sino porque las dimensiones -en comparación con el resto del territorio- cambian aumentando de tal manera que no sabemos si estamos al inicio o al término de algo.

Hablar de Magallanes es evocar una geografía de planos infinitos; eso sí, es más que pampa. Magallanes es un territorio que distingue entre frontera política y frontera geográfica, entre agua y tierra, entre configuración de relieve y tierra. $\mathrm{Si}$ se considera al todo geográfico conocido históricamente como Tierra Magallánica ${ }^{2}$ (Fig. 1), es posible distinguir tres franjas en sentido norte$\operatorname{sur}^{3}$ (Fig. 2):

Franja 1: la zona insular (al poniente del territorio, entre $\operatorname{los} 76^{\circ}$ y $73^{\circ} 50^{\prime}$ '), caracterizada por la presencia del agua como definidora de una estructura de relieve. En esta zona la tierra, por su fragmentación, es acotada y dimensionable. La ocupación se hace imposible apareciendo el mar como el espacio natural ocupable, análogo a una gran quebrada.

$\mathrm{Al}$ oriente de la zona insular aparecen los ventisqueros, sumatoria de ríos de hielo que definen a
3 Este encuadre recupera o evoca el
interés de conquista de los primeros
fundadas conocidos son las ciudades exploradores: la búsqueda del fin del Gamboa en 1584: Nombre de Jesús y mundo, ya seaa través del mar, entre sus Rey Fe lipe. La primera estaba situada serranías o atravesando la pampa. Ver sobre la desembocadura atlántica de Trazado y Territorio: Un estudio de los Estrecho, en el lugar más resguardado tesis de la autora para optar al título edificada al norte de Punta Santa Ana de arquitecto y al grado de Magister en Los dos pueblos fueron habitados por de arquitecto y al grado
Arquitectura PUC, 1997.
Magallanes como un territorio no reconocible vía terrestre desde el norte del país. Los canales aparecen como las principales vías de comunicación.

Franja 2: la zona cordillerana (entre los $73^{\circ} 50^{\prime}$ y $6^{\circ} 80^{\prime}$ ) se caracteriza por la presencia de la cordillera de los Andes, límite boreal del territorio cuyas tierras aledañas se denominan cisandinas, vale decir, situadas al oriente, al revés de lo que ocurre en el resto de Chile. La cordillera rompe la llanura con sus irregularidades, estableciendo una imagen de contrafuertes que configuran espacios reconocibles pero a una escala tal que no es posible hablar ni de cuencas ni de valles. Por esto las entradas de mar adquieren tanta importancia en esta zona: son los valles centralizados, limitados y estáticos.

Franja 3: la zona pampeana (al oriente del territorio, entre los $69^{\circ} 80^{\prime}$ y $66^{\circ}$ ), aparece separada de la franja cordillerana por la presencia del límite fronterizo, que marca la disminución de las pendientes hasta dar paso a la llanura. Lo interesante de esta zona es que en ella se invierte una situación generalizada en el resto de Chile, donde el mar es el plano infinito prioritario; aquí,
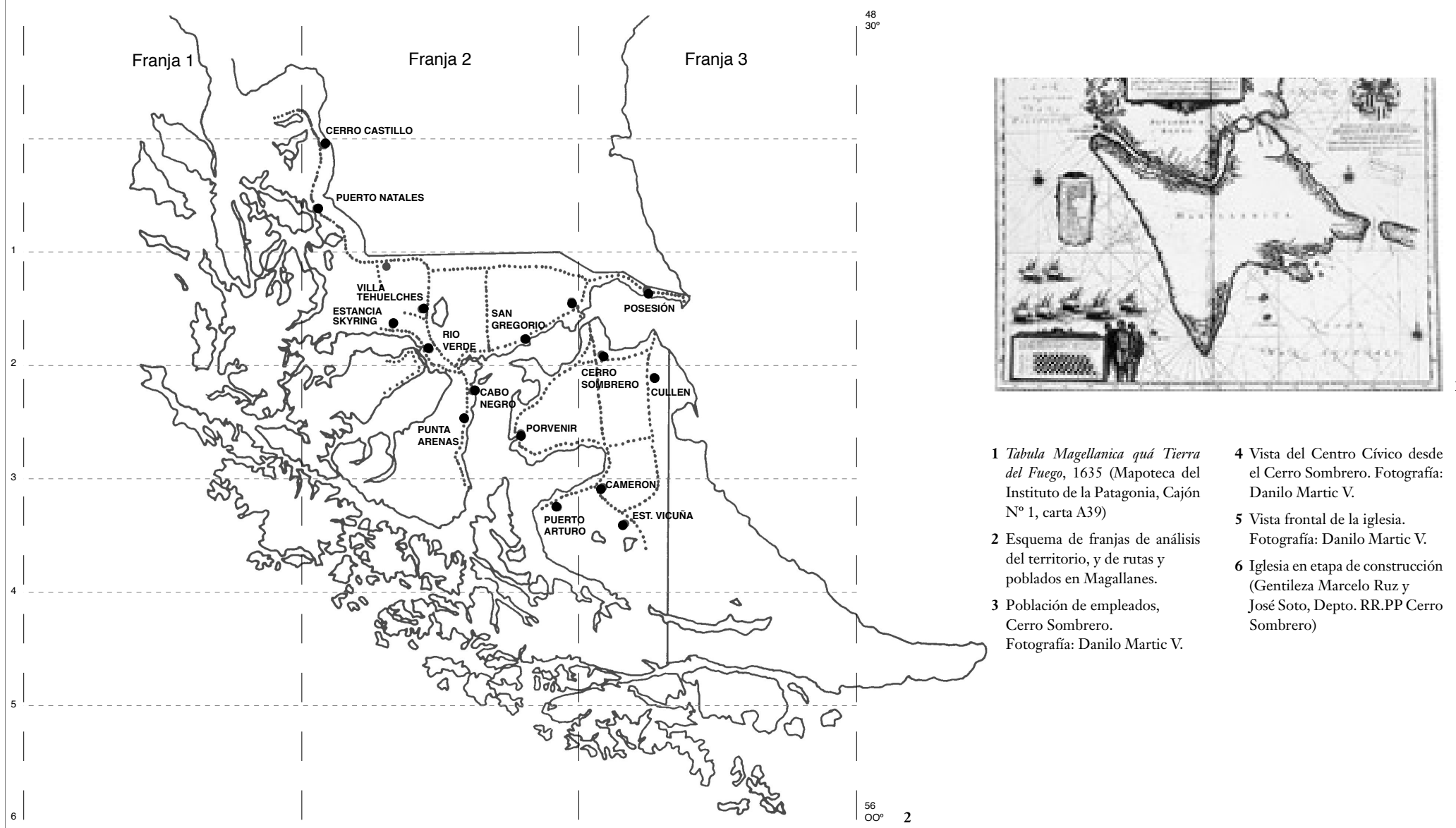

1 Tabula Magellanica quá Tierra del Fuego, 1635 (Mapoteca del Instituto de la Patagonia, Cajón $\mathrm{N}^{\circ} 1$, carta A39)

2 Esquema de franjas de análisis del territorio, y de rutas y poblados en Magallanes.

3 Población de empleados, Cerro Sombrero. Fotografía: Danilo Martic V.
4 Vista del Centro Cívico desde el Cerro Sombrero. Fotografía: Danilo Martic V.

5 Vista frontal de la iglesia. Fotografía: Danilo Martic V.

6 Iglesia en etapa de construcción (Gentileza Marcelo Ruz y José Soto, Depto. RR.PP Cerro Sombrero) 


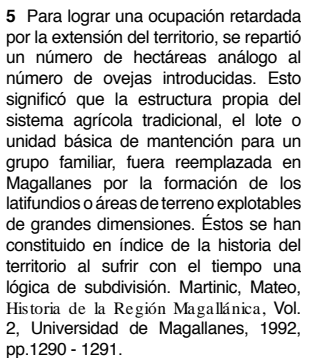

más que un límite físico, la tierra se convierte en horizonte para el resto del territorio.

Magallanes aparece caracterizado entonces por su escala y por la presencia del accidente en forma de sutiles pliegues topográficos que dan origen a una imagen unitaria y continua; un todo geográfico. Sólo después de numerosos intentos se entendió que no bastaba la fundación de una ciudad como punto focal para asegurar la ocupación efectiva ${ }^{4}$. La ocupación se realizó entonces a través de la delimitación del territorio en áreas productivas que no respondieron a un planeamiento, sino más bien a una generación espontánea, paralela a la aparición de los recursos.

Magallanes: una composición entre sectores productivos y áreas geográficas

Los hechos que resumen los modos de ocupación del territorio se pueden resumir en:

La subdivisión predial: En 1920, año de consolidación de la ganadería, los centros de producción (estancias) más importantes eran 17 unidades que abarcaban cerca del $90 \%$ de la superficie habitable de la región. Esta concentración del latifundio $^{5}$ como régimen de explotación, determinó que Magallanes se entendiera como la
7 Antecedentes históricos obtenidos Fue gos, Municipalidad Le Porvenir. 1982 pp. $164-203$; Historia del Petróleo en
Magallanes, Empresa Nacional del Magallanes, Empresa
Petróleo, $2^{2}$ edición, 1993

8 En 1952 inicia su operación la planta de Gasolina de Manantiales, primera consuno de la zona abstecia el uso del gas licuado en el pais. En 1954 entran en funciones la refinería de Concón y el terminal Quintero, que recepciona el crudo que proviene de y 1980 se agregaron a la produción 37 yacimientos petroliferos y 14 campos productoress de gas en una nueva extensión del área productiva. En 1978
se da comienzo a una nueva fase exploratoria con el uso de plataformas submarinas en la boca oriental del Estrecho de Magallanes, el denominado y el 6 de diciembre de 1979 se pusieron en funcionamiento los yacimientos de Ostión y Spiteful, situados a 8 y $16 \mathrm{~km}$ de la costa fueguina, en el área marítima de a Bahia Lomas. En 1988 se encontraban en producción 36 platatormas en 9 crea Sipetrol, Sociedad Internacional Petrolera, filial de ENAP, con el propósito de abordar las actividades de exploración producción de petróleo y gas en el extranjero.

sumatoria de áreas territoriales organizadas por conjuntos de edificaciones, donde se centraba la administración y producción estanciera, y a un conjunto de sitios de pastoreo.

El trazado de las rutas: En la tierra las rutas se fueron conformando por la unión de puntos de ocupación (las estancias y poblados), superponiéndose al territorio donde su geografía lo permite, estableciéndose una red de enlace que permite alimentar a otros puntos que existen entre rutas (estancias menores y puestos). ${ }^{6}$

La actividad petrolera ${ }^{7}$ (Fig. 2): Con el descubrimiento del primer yacimiento comercialmente explotable en Chile, el 29 de diciembre de 1945 en la Isla Grande de Tierra del Fuego, la posteriormente formada Empresa Nacional del Petróleo inició el desarrollo de sus trabajos ${ }^{8}$ en base a la construcción de baterías de recepción (en Punta Catalina), pozos terrestres y pozos en el Estrecho, núcleos de población o campamentos (Cullen ${ }^{9}$, Posesión ${ }^{10}$, Cerro Sombrero ${ }^{11}$ ), terminales de productos (en Cabo Negro, San Gregorio, Isla Percy-Clarencia ${ }^{12}$ ), plantas o centros de procesamiento (Cullen, Posesión, Topping de Gregorio), maestranza de Torres y Barcazas; todas interconectadas por oleoductos, gasoductos
Con 325 habitantes en 1970 , hoy sólo permanecen $20 \mathrm{p}$
régimen de turnos. mento se atienden los yacimientos de área continental. Existen 63 casas para pobladoresyacomodaciones para mas turn

11 En 1965 y producto de la nueva (11 En Registro Civil, Correos, Tenencia de Carabineros, Banco y oficinas mentia cipales, entre otros.
10 Actualmente desde este campa-
12 Ubicados en Bahía Gente Grande objetivo es la transferencia de petróleo crudo, productos limpios y gas licuado a urbano completo estaba al servicio o Eugenio, Las ciudades del salitre, Origenes, 1999, p. 39. 14 Popularmente estos trabajadores $\mathrm{s}$
conocian como $15-5$; con 15 días de trabajo y 5 de descanso en Punta
Arenas.
13 Que "se entendieron como medio de producción donde el conjunto

y poliductos, además de caminos, puertos, aeropuertos y helipuertos propios.

El área de acción de estos establecimientos está concentrada entre la parte norte de Tierra del Fuego (el sector de Punta Dungeness y Posesión) y el área del Estrecho de Magallanes (desde Punta Arenas hacia el nororiente).

Cerro Sombrero y la arquitectura del petróleo

A diferencia de, por ejemplo, las oficinas salitreras ${ }^{13}$, los campamentos petroleros fueron la solución para la instalación definitiva de la población, facilitando la ocupación efectiva de Tierra del Fuego. Con la habilitación de los campamentos Manantiales (1952), Clarencia y Puerto Percy (1953), quedan atrás 10 años de traslado y estadía, sistema característico de trabajo de la zona. ${ }^{14}$

Pero había que formar un centro equidistante de yacimientos y faenas, que permitiera manejar técnica y administrativamente la totalidad de la Isla. Así se gestó Cerro Sombrero, el caso más relevante de la arquitectura del petróleo en Magallanes.

Cerro Sombrero ${ }^{15}$ inició su existencia como
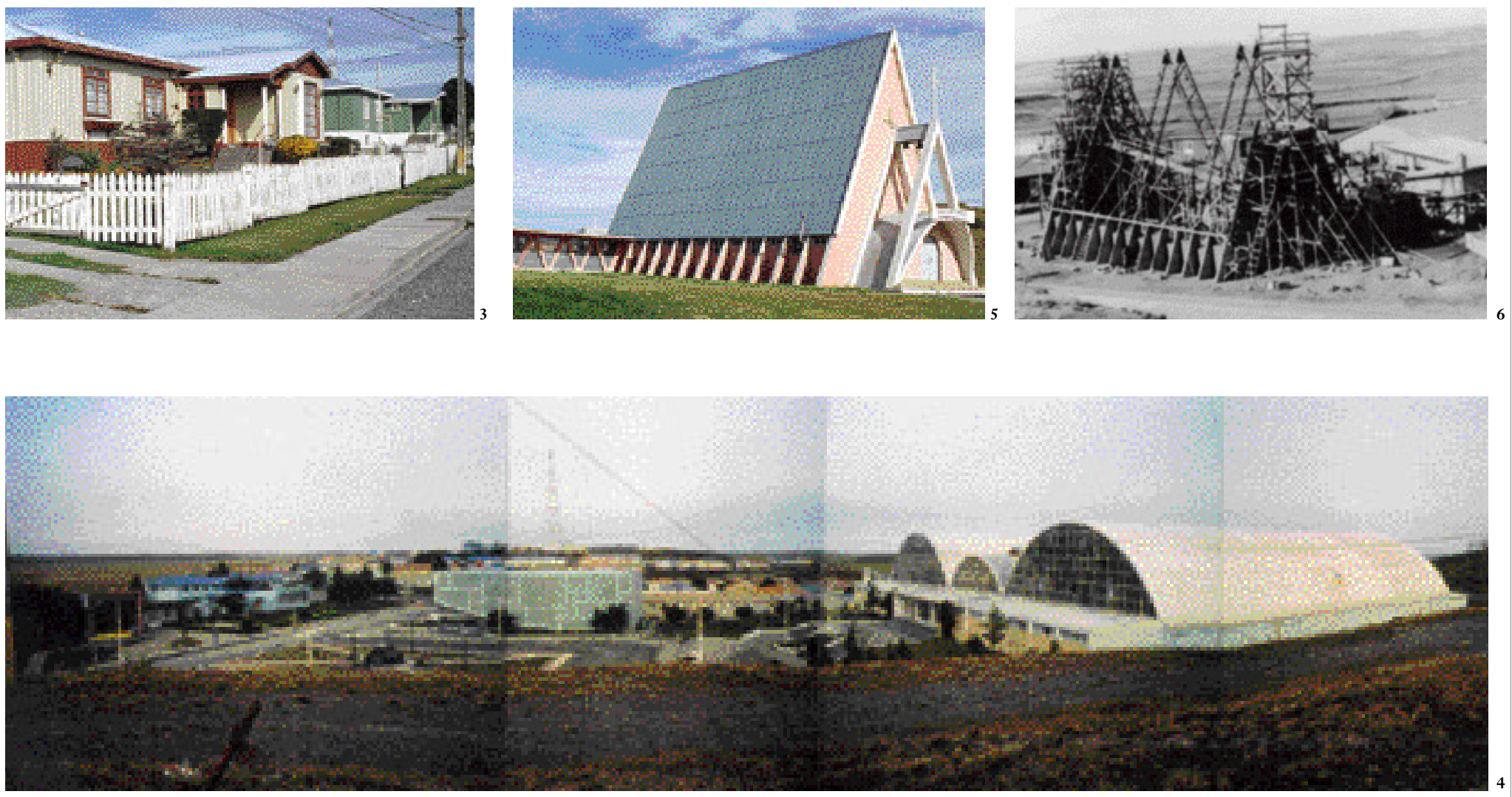
campamento en 1949, con la instalación de un equipo de trabajadores en las inmediaciones del río Side, pero los constantes desbordes del mismo provocaron la construcción de un poblado que asegurara condiciones de habitabilidad para sus trabajadores. En 1957 se instalaron en torno al cerro Side (con forma de sombrero) las primeras casas, dando paso en 1958 y hasta 1961 a la edificación del conjunto, proyectado para más de 100 familias. El poblado se organiza en 5 sectores: la población de empleados, el centro cívico, la población de choferes, la población de obreros y el sector industrial.

La población de empleados (Fig. 3) está constituida por 31 viviendas distribuidas en tres manzanas. En total $3.640 \mathrm{~m} 2$ distribuidos en terrenos promedio de $928 \mathrm{~m} 2$. Las casas, de un piso y $13.16 \mathrm{~m}$ de frente, poseen en $150 \mathrm{~m} 2 \mathrm{un}$ programa que cuenta con: porche, vestíbulo, ball, 3 dormitorios, 1 baño, living, comedor, cocina y despensa. Se suman $94 \mathrm{~m} 2$ en el zócalo (de muros de concreto de $30 \mathrm{~cm}$ ): lavadero, dormitorio de servicio y sala de juegos. La estructura de la casa, en base a pilares de roble ensamblados de 3 " x 4", está forrada exteriormente con raulí machihembrado de 1" x 6 " y revestida a su vez con planchas acanaladas. En el interior la casa está totalmente revestida en pino estriado de 1 " $\mathrm{x}$ 8". Cabe señalar que todas las viviendas contaban con agua potable, alcantarillado, electricidad, gas natural y teléfono.

El centro cívico (Fig. 4) es la suma de: el terreno de la iglesia en el sur oriente (alvéolo de $1.990 \mathrm{~m} 2$ ), el polígono irregular de $5.500 \mathrm{~m} 2$ que contiene a la loma que da nombre al pueblo (Escuela, Policlínico, Cine y Centro Deportivo), y el rectángulo de la pulpería y casino (hacia el sur).

La iglesia (Figs. 5 y 6) es una nave rectangular de $20.85 \times 9.45 \mathrm{~m}$ a la que se suma un atrio de $3 \times 6.80 \mathrm{~m}$. La techumbre -de $14 \mathrm{~m}$ de alto- llega a tierra a través de 14 contrafuertes laterales triangulares de hormigón, situados a una distancia de $1.40 \mathrm{~m}$. Caracteriza a la iglesia su tijeral triangular de $\mathrm{Fe}$ armado de $40 \mathrm{~cm}$, forrado interiormente con madera de 1" x 6" y exteriormente con $\mathrm{Fe}$ galvanizado. Adosada a la iglesia se encuentra la casa parroquial, que a la larga será usada como internado, con una planta en "L" de 140 m2.
5Para llegar al lugar desde Pun Arenas hay tres altemativas: viajar sábado (en dos horarios) hasta Porveni y desde ahi dirigirse por camino de egar: realizar un cruce en barcaza desde el sector Tres Puentes, en las inmediaciones de la capital regiona, que dura aproximadamente dos horas desembarca en Bahía Chilota, ys inmediaciones de Porvenir, y desde

tierra los mismos $125 \mathrm{~km}$ ya indicados. Cabe señalar que este cruce está por lo que existen días de suspensión durante todo el año. Finalmente, tercera opción es dirigirse $166 \mathrm{~km}$ por Primera Angostura donde hay cruces diarios de 20 min de duración. En este caso, una vez en la Isla, hay que dirigirse por camino de tierra $43 \mathrm{~km}$ al sur hasta
El complejo deportivo (Fig. 7) preside el centro antecedido por una plaza de $47.5 \times 41 \mathrm{~m}$, y por una escalera monumental de acceso que llega 8 m más arriba del resto del pueblo y 5 más abajo del emplazamiento del observatorio, a $4 \mathrm{~m}$ de la cumbre. El complejo alberga programas diversos organizados en naves parabólicas de estructuras metálicas independientes; la piscina y el gimnasio tienen $24 \mathrm{~m}$ de frente y 10 de alto. El solarium, de $15 \mathrm{~m}$ de frente y $7.20 \mathrm{~m}$ de alto, está organizado en base a tabletas circulares de concreto de distintos tamaños agrupadas a modo de lotos que contienen fuentes de agua, vegetación y mobiliario.

El cine, para 537 butacas y construido en hormigón, es un paralelepípedo regular de 150 x $31 \mathrm{~m}$, caracterizado por su muro poniente que recoge la pendiente del teatro y que se constituyó en mural de colores característicos y visible desde las afueras. Cierra este conjunto hacia el poniente la Escuela Básica F-41 con $1.220 \mathrm{~m} 2$ y el Policlínico, de $800 \mathrm{~m} 2$.

La pulpería (Fig. 8) es un volumen rectangular de $25.9 \times 10 \mathrm{~m}$, caracterizado por su frente transparente, invernadero y vitrina de exhibición
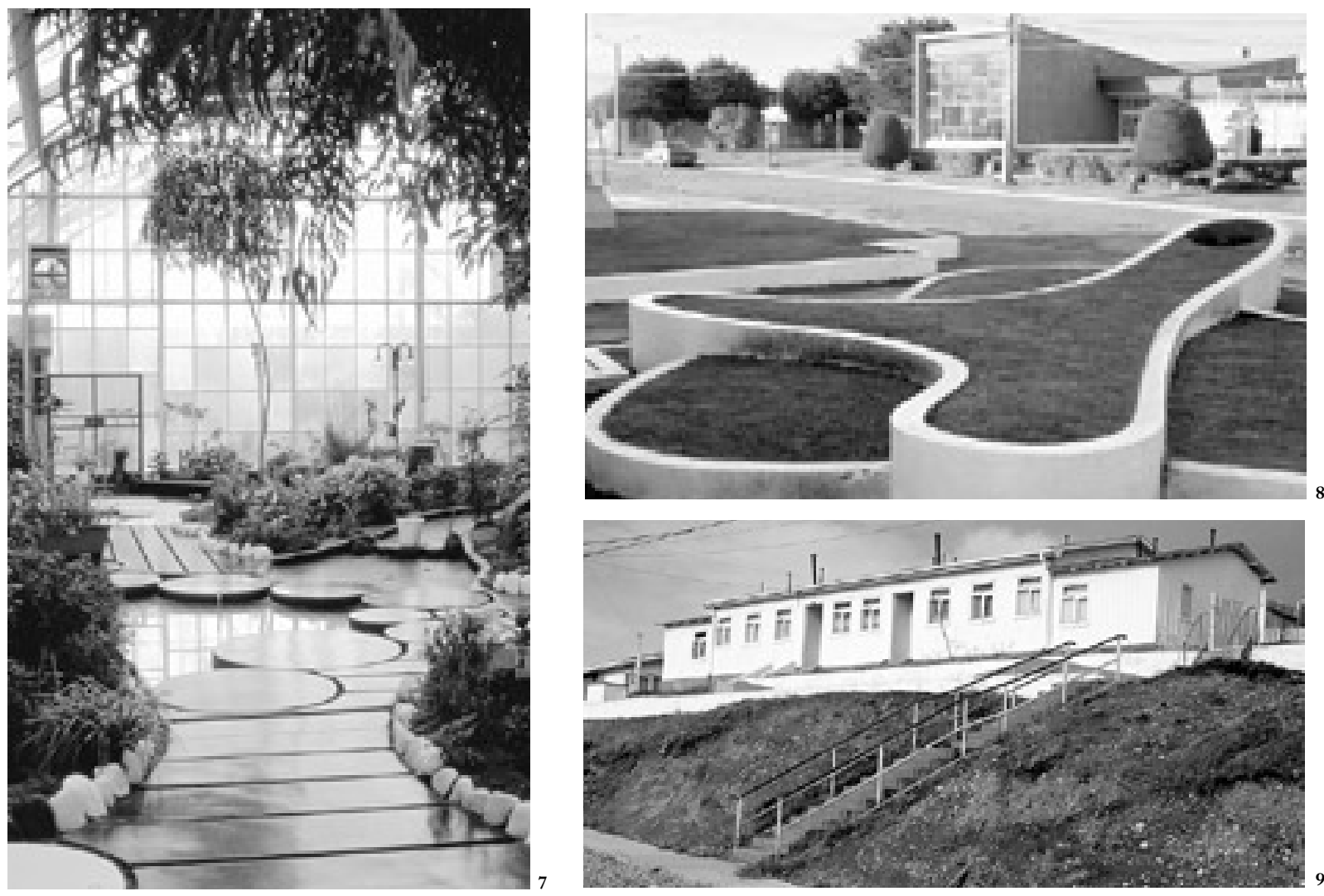

\author{
7 Solarium. Fotografía: \\ Danilo Martic V \\ 8 Pulpería. Fotografía: \\ Danilo Martic V. \\ 9 Población de choferes. \\ Fotografí: Danilo Martic V.
}

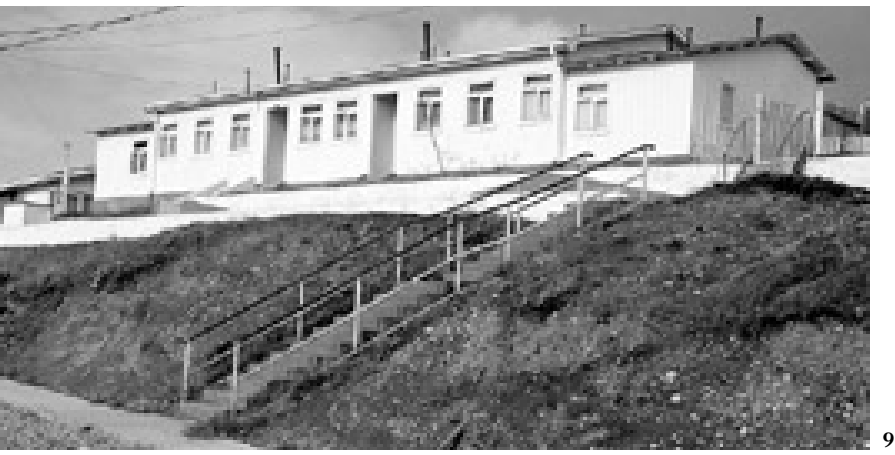


16 Integrado por patio bodega y bodegas de servicio, pañol producción, talleres

17“(.) Unidad urbana capaz do trabajo, a vivienda los servicios (...) Esta condición de autonomía fue consolidada por la presencia de tres elementos: las astalaciones industriales (...); los equie salud, adquisición de productos básicos, educación, esparcimiento otras; $y$ las viviendas, para el alojamiento los obreros, empleados, técnicos a la vez. Hacia el sur se adosa el volumen de servicios de $20 \times 8.40 \mathrm{~m}$ y hacia el norte la galería comercial de $10.40 \times 16.6 \mathrm{~m}$. El casino, que ocupa $1.100 \mathrm{~m} 2$, es una planta en " $\mathrm{H}$ " con remates abocinados.

A la población de choferes (Fig. 9), de 21 viviendas pareadas de $76.5 \mathrm{~m} 2$, se accede a través de escaleras frontales. Son 3 manzanas rectangulares que crecen en largo para rodear a un montículo existente y constituir el límite poniente del conjunto. El resto del conjunto está delimitado por cierros metálicos y cortavientos de madera.

La población de obreros son 3 manzanas con 2 tipos de vivienda: 7 casas (frente a la Escuela y Policlínico) para los $15-5$ y el resto, 46 viviendas pareadas en terrenos de $390 \mathrm{~m} 2$. Con una superficie de $97.8 \mathrm{~m} 2$, contienen en un piso el mismo programa que las casas para empleados. La estructura, a diferencia de éstas, es de bloques de concreto estucados al interior, con piso entablado de roble de 2" x 2" y cielo de madera en pino machihembrado de 1 " x 3 ".

El sector industrial de $109.687 \mathrm{~m} 2$ encabeza el acceso al poblado ${ }^{16}$.
De acuerdo a lo descrito, no cabe duda que Cerro Sombrero pone en evidencia la estrategia de fundación de patrones industriales ${ }^{17}$ en un territorio que, como hemos visto, no otorga señales de ocupación. Pero es interesante constatar que, al mismo tiempo, experimenta la transferencia de ideas, referentes y modelos de los principales centros de arquitectura moderna de las décadas de los años 1940 y 1950, período de su construcción. Las formas exteriores de los edificios que configuran el centro cívico principal (iglesia, cine, centro deportivo y pulpería), recuerdan parte del historial de la arquitectura brasileña, específicamente la de Oscar Niemeyer para la urbanización del lago de Pampulha. Las naves parabólicas de la iglesia de San Francisco de Asís (1943), los aleros del Casino (1942) y la inclinación del techo del club de Yates (1942), son todos elementos visibles en Sombrero y pueden encontrarse también en la Biblioteca de la Facultad de Ingeniería en la Ciudad Universitaria de Carlos Villanueva (fig. 10); en el edificio, escuela y gimnasio de Pedregulho (1950-1952) o en el Teatro comunitario del distrito de Marecharl Hermes (1950), ambos de Alfonso Reidy. La construcción de este vocabulario se adaptó al del acero usado como material estructural, tecnología que se supone propia de la empresa norteamericana que ejecutó el proyecto.

Las experiencias de Pampulha (Fig. 11) y Cerro Sombrero tienen otro importante punto en común: ambos conjuntos surgen como pequeñas localidades a cierta distancia de una ciudad mayor (Bello Horizonte y Punta Arenas respectivamente); ambos se originan por un interés económico específico (promover el turismo y cimentar el petróleo, respectivamente); y sobre todo, ambos fueron realizados bajo la idea comúnmente aceptada por la arquitectura moderna del proyecto de fundación de una ciudad completa -ex novo. Tanto Pampulha como Cerro Sombrero fueron construidos donde no había nada.

No extrañaría entonces que el arquitecto de la ENAP Julio Ríos Boettiger (iglesia) y los especialmente contratados Monckeberg, Echevarría y Briones; Jorge Searle; Bolton, Larraín, Prieto; Juan Echenique y José Cruz (todos firmantes en el cine, supermercado y centro deportivo) miraran con atención el caso de Pampulha, entre otros, permitiendo con ello configurar la construcción de arquitectura moderna más al sur del mundo. ARQ
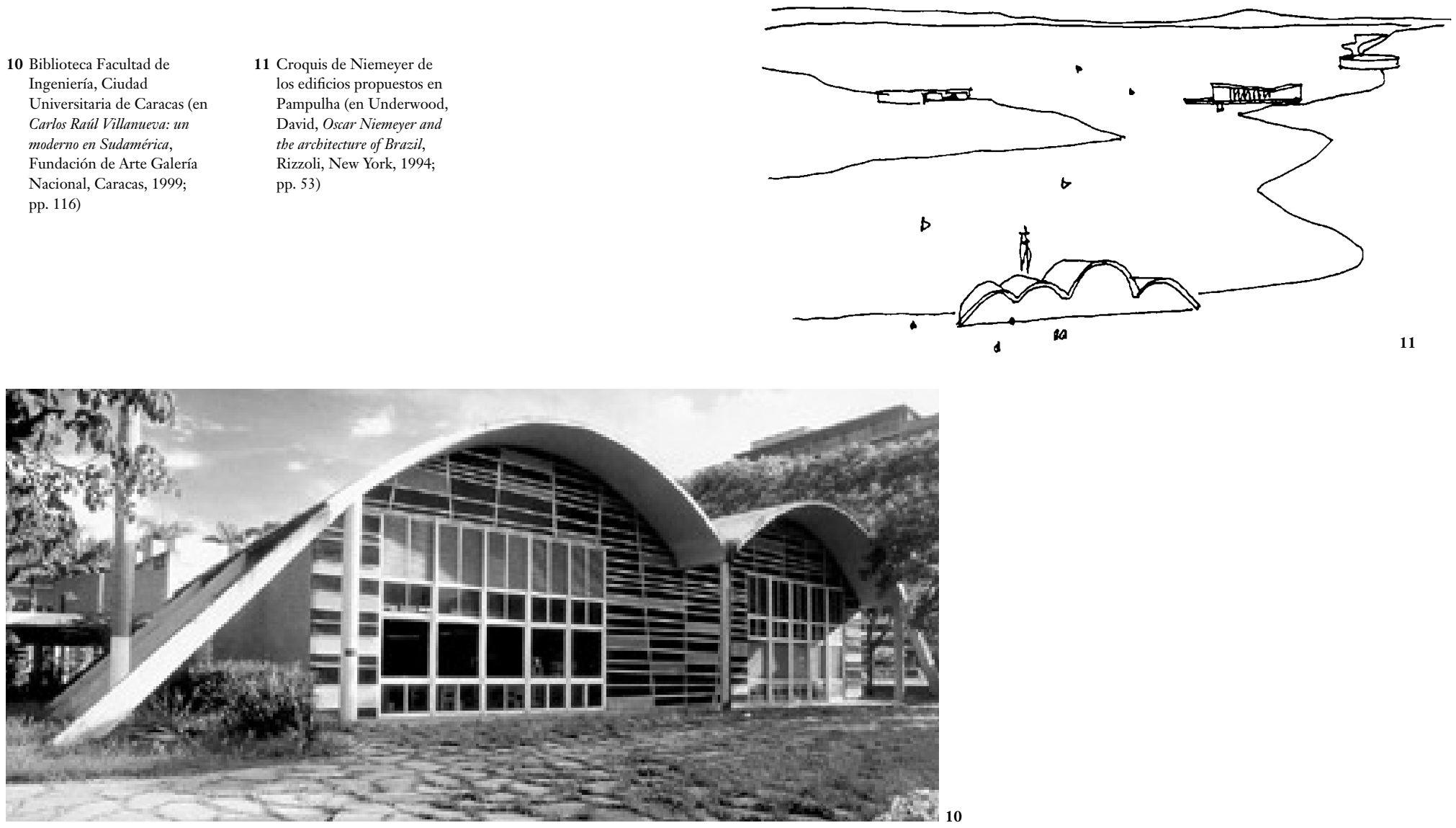

11 Croquis de Niemeyer de los edificios propuestos en Pampulha (en Underwood, David, Oscar Niemeyer and the architecture of Brazil, Rizzoli, New York, 1994; pp. 53) 